$\xi=-1$

\title{
Optimal Block Diagonalization precoding scheme for MU-MIMO Downlink channel Using Particle Swarm Optimization
}

\author{
Shivangini Saxena ${ }^{1 *}$, Dr. R.P. Singh ${ }^{2}$ \\ ${ }^{I}$ (Research Scholar, Department of Electronics and Communication, Sri Satya Sai University of Technology \& Medical Sciences, \\ Pachama, Sehore, (M.P.), India.) \\ ${ }_{2}^{2}$ (Professor, Sri Satya Sai University of Technology \& Medical Sciences, Pachama, Sehore, (M.P.), India.) \\ *Email: enggshivangini2006@yahoo.co.in
}

\begin{abstract}
As wireless communication turns out to be more common, the interest for higher rates of data transfer and continuous availability is expanding. Future wireless systems are provisioned to be very heterogeneous and interconnected. Higher data rates and Quality of Service (Qos) are two major expectations from any wireless technology. Fading is the main phenomenon which restricts the realization of Qos demand and higher data rates in wireless technologies. Fading is caused by obstacles in signal path which degrades the received signal's quality. To mitigate the impact of fading on communication system the application of precoding techniques can be used. In this regard, this paper presents optimization of Block-Diagonalization (BD) based linear precoding scheme for multi-user multiple-input multiple output (MU-MIMO) systems. Simulation environment consists of a MIMO downlink scenario where a single base station (BS) with $N_{t}$ antennas transmits to K receivers each with $N_{r}$ antenna. The application of Particle Swarm Optimization (PSO) is used to find the optimal number of received antennas so as to reduce system complexity while maintaining Bit Error Rate (BER) performance of the system. MATLAB based simulation scenario is presented and evaluated over Rayleigh fading environment. Simulation results validate that the performance of Block- Diagonalization scheme can be improved up to 5dB with the application of Particle Swarm Optimization technique.
\end{abstract}

Keywords: Block-Diagonalization (BD), Multi-User Multiple-Input Multiple-Output (MU-MIMO) system, Optimized Precoding, Particle-Swarm Optimization

(PSO).

\section{Introduction}

The demand for increasingly high transmission rates from wireless communication systems and new mobile applications has made it necessary to find more advanced transmission methods capable of improving the performance of the system. A possible solution is use of so-called MIMO systems that, through the use of multiple antennas in the transmitter and / or receiver, allow to increase the capacity of the system without the need to increase neither the bandwidth nor the transmission power [1]. MIMO systems take advantage of the spatial dimension to distribute the total power between the different antennas and thus achieve array gain (improves the spectral efficiency of the system) or diversity gain (improves the reliability of the link) [2]. The performance of MIMO systems is restricted by the number of antennas deployed at transmitter and receiver ends. Furthermore, the system complexity of MIMO systems also increases with the number of antennas deployed. The performance of MIMO systems can be enhanced if the channel state information (CSI) can be made available at transmitter end. It is then possible to utilize CSI to optimize overall transmission by anticipating the effect of the channel [3]. This optimization is made via a precoder at transmitter end, and a decoder at receiver end via mostly linear transformations. This technique provides MIMO systems with an additional degree of freedom choosing an optimization strategy. The basic idea of precod- ing is to use a form of knowledge of the channel to the transmitter to customize the signals transmitted to the proper structure of the matrix channel. Precoding improves the probability of error, the transmission rate and therefore the capacity of the system by a more efficient sharing of the channel with the different users, it also allows the suppression of interference and the occurrence reduces the complexity of the receiver [4]. In information theory, it is well known that the optimal strategy for achieving the sum of the capabilities of a downlink MU-MIMO system is the Adjacent information coding (DPC - Dirty Paper Coding) [5]. In this theory, the capacity of a channel where the transmitter knows a priori the interfering signal is the same only if there was no interference. Therefore, precoding can be applied considering this knowledge in order to remove interference at the level of the issuer. However, the implementation of this technique remains impractical in because of the high complexity of coding and decoding [6]. For suboptimal performance with low complexity, several types of linear and nonlinear precoders have been proposed [7-10]. An example of such precoder is the Tomlinson-Harashima precoder (THP) [7], [8]. Tomlinson-Harashima precoder is the most popular nonlinear precoder. It is basedon the principle of equalization of the Decision-Feedback Equalization (DFE). THP provides proven performance gain in MU-MIMO scenario but its deployment in real-time systems is restricted due to its higher complexity. Moreover, the performance of THP is sensitive to the inaccuracy of the CSIT [11].For applications requiring low complexity, Zero Forc- 
ing (ZF) and Minimum Mean Square Error (MMSE) precoding techniques can be used. These methods are known as channel inversion methods [9]. Precoding based on the ZF algorithm can completely suppress multi-user interference (MUI). However, it causes noise amplification when precoding vectors are not standardized. On the other hand, precoding based on the MMSE algorithm surpasses the $\mathrm{ZF}$ algorithm because it makes a compromise between noise and deletion interference. Nevertheless, it suffers a loss of performance when it tries to attenuate the interference between two antennas close together [12]. To overcome this problem, the SMMSE (Successive Minimum Mean Square Error) calculates successively the columns of the precoding matrix for each of the reception antennas separately [13]. These channel inversion methods are not effective when the receiver is equipped with multiple antennas. In such case, Block Diagonalization (BD) precoding technique comes into existence. Block Diagonalization technique cancels the interference by breaking MU-MIMO into several parallel and orthogonal SU-MIMO channels using Singular Value Decomposition (SVD) [14]. Block Diagonalization scheme is interesting in the case where the receiver is equipped with an antenna array. Moreover, in terms of performance, it is better than the channel inversion methods and it is capable of eliminating multi-user interference (MUI) completely. Whereas, it leaves the receiver with the responsibility of separating the data streams that are sent to it and it requires more antennas on the transmitter than the number of antennas at receiver for all users. Recent researches on Block-Diagonalization precoding are focused towards the performance optimization through application of soft computing techniques such as Genetic Algorithm (GA), Particle-Swarm Optimization (PSO) etc. This paper presents the optimization of Block-Diagonalization scheme through PSO algorithm. The optimization is done for finding optimal number of receiver antennas so as to provide minimum Bit Error Rate (BER). Rest of this paper is organized as follows: Section-II discusses related work in field of MU-MIMO precoding. In Section-III, proposed system model for downlink MIMO system is presented, results and discussion is provided in section-IV, finally section- $\mathrm{V}$ concludes this paper.

\section{Related Work}

Performance enhancement through application of precoding technique is interesting area of research since last decade. Several researchers have contributed towards this field. F. Wang and M. E. Bialkowski [14] have presented two antenna selection techniques namely norm-based and project-maximization based to improve the performance of MU-MIMO system with Block Diagonalization precoding scheme. Authors have concluded that the application of antenna selection schemes improves channel capacity while reducing complexity and cost of the systems. M. Naeem and D.C. Lee [15] have presented an optimised joint user scheduling and receive antenna selection algorithm based on Binary Particle Swarm Optimization (BPSO) approach to improve the performance of MU-MIMO system.W. Ni and X. Dong have considered case of MIMO downlink scenario and presented hybrid block diagonalization scheme to improve the capacity performance of conventional $\mathrm{BD}$ scheme. On comparing performance, proposed hybrid-BD scheme is found to be less complex and more spectral efficient as compared to conventional BD scheme[16].X Zhu et al have proposed a combination of soft pilot reuse (SPR) and multicell block diagonalization scheme to improve the quality-ofservice $(\mathrm{QoS})$ performance for edge users in MU-MIMO system [17]. To facilitate hardware implementation of massive MUMIMO system, J.C. Chen [18] et al proposed a low peak-toaverage power ratio (PAPR) precoding scheme based on approximate message passing scheme. The proposed scheme provides better performance in terms of multi-user interference (MUI) cancellation while having low complexity. V.F. Crâş̧mariu et al [19] presented a QR-decomposition based approach for optimizing the performance of BD in MU-MIMO systems. ElinaNayebi et al have considered Cell-free Massive MIMO systems to evaluate the performance of linear precoding schemes. The considered scenario consists of a number of single antenna access points (APs), which are connected to a centralised network controller. The authors have shown the impact of zero forcing (ZF) precoder with MaxMin power control to the overall throughput achieved at several users and concluded that ZF precoding outperforms conventional conjugate beamforming algorithm [20]. M. Ge and D. M. Blough have addressed the problem of user selection on the performance of multi-user MIMO system and presented a pairing-and-binary tree-based user selection algorithm (PBUS) for optimal user selection. Authors have concluded that the proposed scheme provides better sum rate performance while having low computational complexity [21]. The application of evolutionary algorithms such as GA and PSO can be used to optimize the performance of MIMO system is presented by N. sindhwani et al [22]. Authors have proposed an optimal transmit antenna subset selection scheme based on PSO algorithm to achieve optimal channel capacity while maintaining lowest BER performance. The application of Riemannian manifold optimization (RMO) to solve the problem of multi-user interference (MUI) in constant envelope (CE) precoding scheme [23]. B. Makki et al proposed Genetic Algorithm (GA) based antenna selection scheme for LargeMIMO systems with finite antenna arrangement. The proposed scheme is evaluated for different cases of CSI and precoding arrangements [24].

\section{Proposed Methodology}

\section{MIMO Downlink System Model}

Let us consider a downlink Multi-User MIMO scenario where a single Base Station (BS) is serving $\mathrm{K}$ users via $N_{t}$ antennas, Also each user is equipped with $N_{r}$ antennas. The considered arrangement is shown in Fig-1. At the reception, the signal received at the $K^{t h}$ user can be expressedby the following equation:

$$
y_{k}=H_{k} x+n_{k}, \quad k=1,2,3 \ldots K
$$

Where $H_{k} \in C^{N_{r} \times N_{t}}$ denotes the matrix of channel coefficients of the $k^{\text {th }}$ user $x \in C^{N_{t}}$ represents thesignaltransmitted by the BS and $n_{k} \in C^{N_{r}}$ is Gaussian white additive noise of $N_{0}$ variance and zero mean.

It is assumed for simulation that the channel is quasi-static in nature i.e. channel states do not vary within a frame of $\mathrm{N}$ symbols, but can change from one frame to another [25]. At the level of each user, it is assumed that the knowledge of the channel is perfect and instantaneous. And, it is not necessary for each user to know the channels of other users.
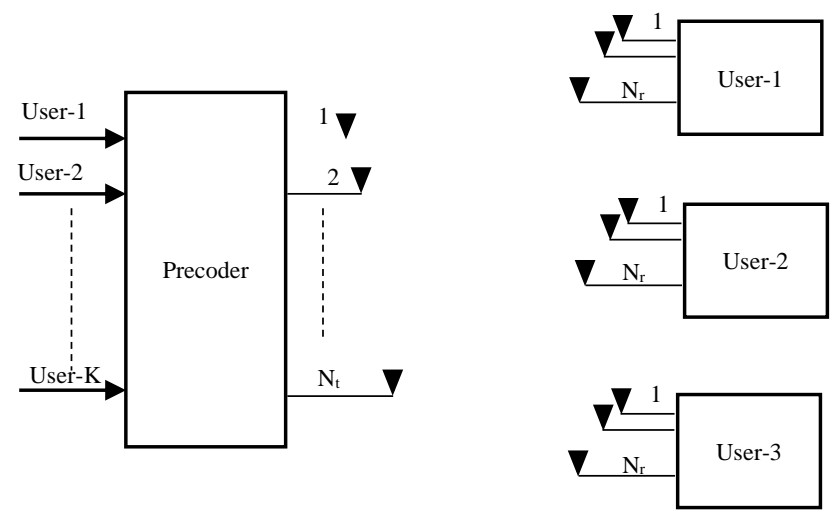

Fig. 1: MIMO Downlink System Model

This research work focuses on liner precoding, therefore, the transmitted signal, $x$, can be given as follows: 


$$
x=\sum_{k=1}^{K} w_{k} S_{k},
$$

Where $w_{k} \in C^{N_{t}}$ presents the precoding vector of $k^{t h}$ user. $S_{k}$ denotes the symbolof information to be transmitted to $k^{\text {th }}$ user.In this case, the signal received at the $k^{t h}$ user can be expressedas follows:

$$
y_{k}=\sqrt{p_{k}} H_{k} w_{k} S_{k}+\sum_{i=1, i \neq k}^{K} \sqrt{p_{i}} H_{k} w_{i} S_{i}+n_{k},
$$

The first part of equation (3) represents the desired signal, while the second part is the interference caused at $k^{\text {th }}$ user due to other users and the last part represents the noise present at $k^{\text {th }}$ user. Furthermore, it is assumed that the BS transmits QPSK symbols (Quadrature Phase-Shift Keying) with a total power $P_{0}$ with following constraints [26]:

$$
\begin{gathered}
E\left[\left\|W_{s}\right\|_{2}^{2}\right]=\operatorname{tr}\left(W_{s S^{H}} W^{H}\right) \leq P_{0} \\
P_{0}=\sum_{k=1}^{K} p_{k}
\end{gathered}
$$

Where $W=\left[w_{1} \ldots w_{k}\right]$ represents the precoding matrix, $S=$ $\left[s_{1} \ldots s_{k}\right]$ is the signalto be transmitted and $p_{k}$ is the portion of transmit power available at $k^{\text {th }}$ user.It is important to mention that this power is considered constant and identical for each user in order to eliminate its effect on the performance of proposed system. With Block Diagonalization precoding scheme, the main idea is obtain precoding vector $w_{l}$ such that, interference from all other users at $k^{t h}$ user must be zero. i.e.

$$
H_{k} w_{l}=0 . \text { for all } l \neq k, 1 \leq l, k \leq K
$$

To satisfy the constraint given in eq. (5), orthogonal channel matrix $\widetilde{H}_{k}$ for all users except $k^{t h}$ user can be given as:

$$
\widetilde{H}_{k}=\left[H_{1}^{T}, \ldots, H_{k-1}^{T}, H_{k+1}^{T}, \ldots H_{k,}^{T}\right]^{T}
$$

To satisfy constraint of zero-interference from other users, $w_{k}$ must lie in the null space of $\widetilde{H}_{k}$. Applying singular value decomposition to the channel matrix $\widetilde{H}_{k}$, resulting matrix can be given as:

$$
\widehat{H}_{k}=U_{k}\left[\Sigma_{\mathrm{k}} 0\right]\left[V_{k}^{(1)} V_{k}^{(0)}\right]^{H}
$$

In Eq. (7), $\Sigma_{k}$ represents a diagonal matrix with entries equals all non-negative singular values $\widehat{H}_{k}$. The dimension of $\Sigma_{k}$ depends upon the rank of matrix $\widehat{H}_{k}$. Furthermore, $V_{k}^{(0)}$ and $V_{k}^{(1)}$ represents vectors corresponding to zero singular values and non-zero singular values respectively. On multiplying $\widehat{H}_{k}$ with $V_{k}^{(0)}$, we get,

$$
\widehat{H}_{k} V_{k}^{(0)}=0
$$

From Equation (8), it can be seen that $V_{k}^{(0)}$ is in the null space of $\widehat{H}_{k}$, that is to say namely, when a signal istransmitted in the direction of $V_{k}^{(0)}$, all but the $k^{\text {th }}$ user receives no signal at all. Thus, the precoding matrix for $k^{t h}$ user can be represented by:

$$
w_{k}=V_{k}^{(0)}
$$

Since Size of $V_{k}^{(0)}$ depends on size of $\widehat{H}_{k}$, we can manipulate the size of $\widehat{H}_{k}$ by setting the number of receiving antennas $N_{R}$ for each user.

To find an optimal value of $N_{R}$ an objective function can be drawn as:

$$
\min f\left(\widehat{H}_{k}\right)=\left|H_{k} w_{k}\right|
$$

Where,

$$
\widehat{H}_{k}=f\left(\dot{N}_{r}\right)
$$

$$
\dot{N}_{R}=\operatorname{PSO}\left(N_{R}\right)
$$

\section{Simulation Results}

This research work is focused towards optimization of BlockDiagonalization for optimal number of receiver antennas using PSO algorithm. The simulation scenario consists of MIMO downlink system where single BS is transmitting to several users. Simulation scenario is developed using MATLAB (2014a) with simulation parameters given as:

Table 1: Simulation Parameters

\begin{tabular}{|l|l|}
\hline Number of transmitter antennas $\left(N_{t}\right)$ & 6 \\
\hline Number of Receiver antennas $\left(N_{r}\right)$ & {$[1-10]$ (to be Found by PSO) } \\
\hline Number of Users $(K)$ & 3 \\
\hline Fading Scenario & Rayleigh Fading \\
\hline SNR Range & $-10 \mathrm{~dB}$ to $20 \mathrm{~dB}$ \\
\hline Modulation Scheme & m-PSK \\
\hline Equalizer & Zero Forcing \\
\hline
\end{tabular}

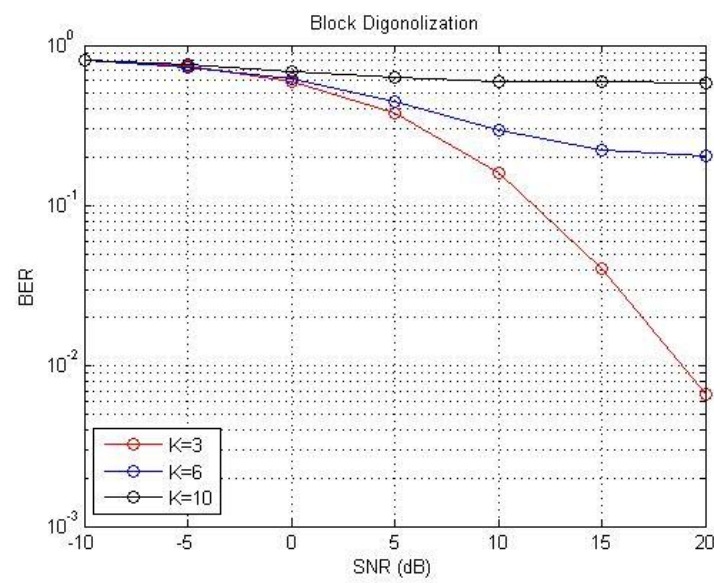

(a)

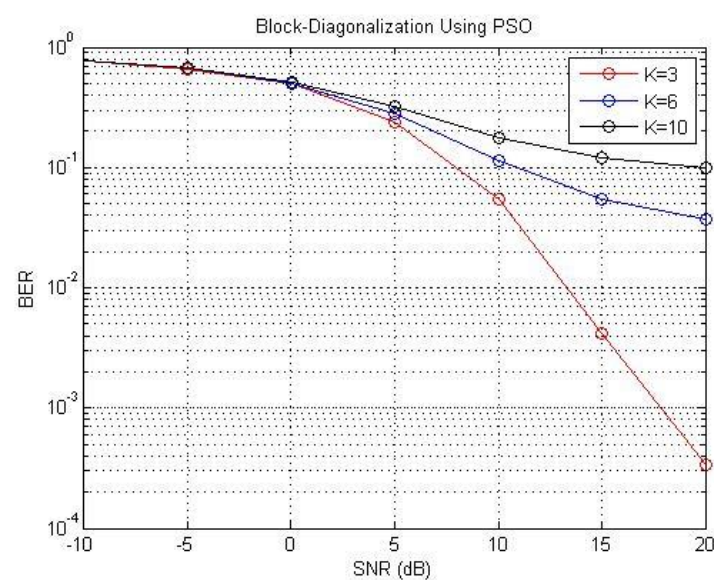

(b)

Fig. 2: BER performance analysis for BD and BD-PSO for Different Number of User Present

Bit error rate comparison of Block-Diagonalization precoding and Optimized Block-Diagonalization scheme for Zero forcing equalizer for different number of users is presented in Fig. 2. On observing above results it can be seen that for 6 users BD scheme achieves error rate of order $10^{-1}$ at $15 \mathrm{~dB}$ SNR, while the same level is achieved in case of PSO-BD at 10dB SNR. Clearly the application of PSO with BD scheme provides SNR improvement up to $5 \mathrm{~dB}$ for the given case.

The value of $\dot{N}_{r}$ can be found as, 


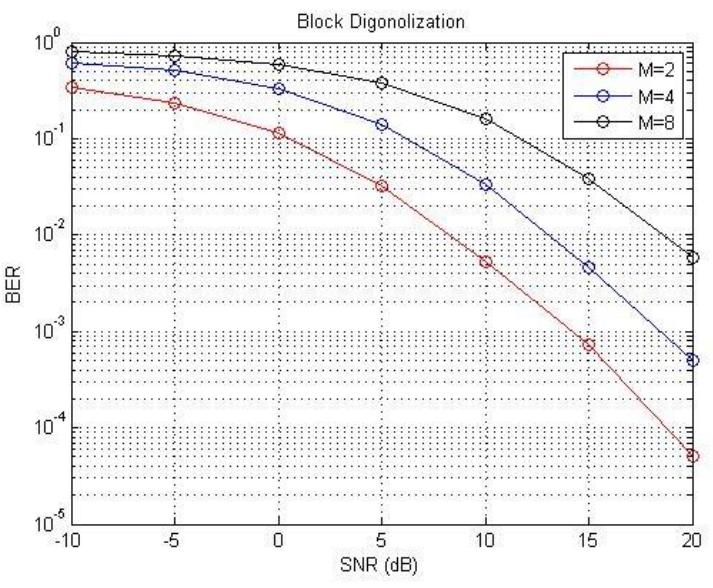

(a)

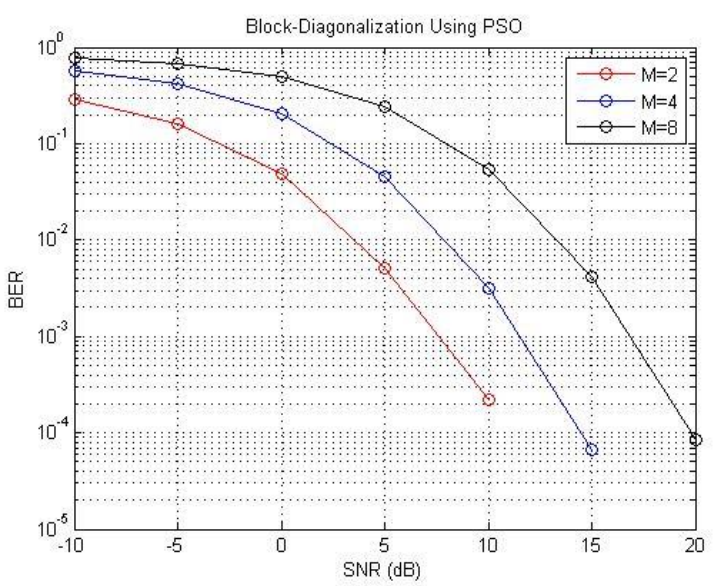

(b)

Fig. 3: BER curve for BD and PSO optimized BD for different modulation schemes

Bit error rate comparison of Block-Diagonalization precoding and Optimized Block-Diagonalization scheme for Zero forcing equalizer for different modulation schemes is presented in Fig. 3. On observing above results it can be seen that for BPSK scheme $(M=2)$ the minimum achievable error rate of order of $10^{-4}$ at $20 \mathrm{~dB}$ SNR while PSO-BD scheme provides minimum error rate of $10^{-3}$ at $10 \mathrm{~dB}$ SNR and error free transmission is obtained after $10 \mathrm{~dB}$ SNR level. Clearly PSO-BD outperforms conventional BD scheme for the considered case. Bit error rate comparison of Block-Diagonalization precoding and Optimized BlockDiagonalization scheme for Zero forcing equalizer for QPSK modulation schemes is presented in Fig. 4. On observing above results it can be seen that the minimum achievable error rate of order of $10^{-3}$ at $20 \mathrm{~dB}$ SNR while PSO-BD scheme provides minimum error rate of $10^{-4}$ at $15 \mathrm{~dB}$ SNR and error free transmission is obtained after 15dB SNR level. Clearly PSO-BD outperforms conventional $\mathrm{BD}$ scheme for the considered case.

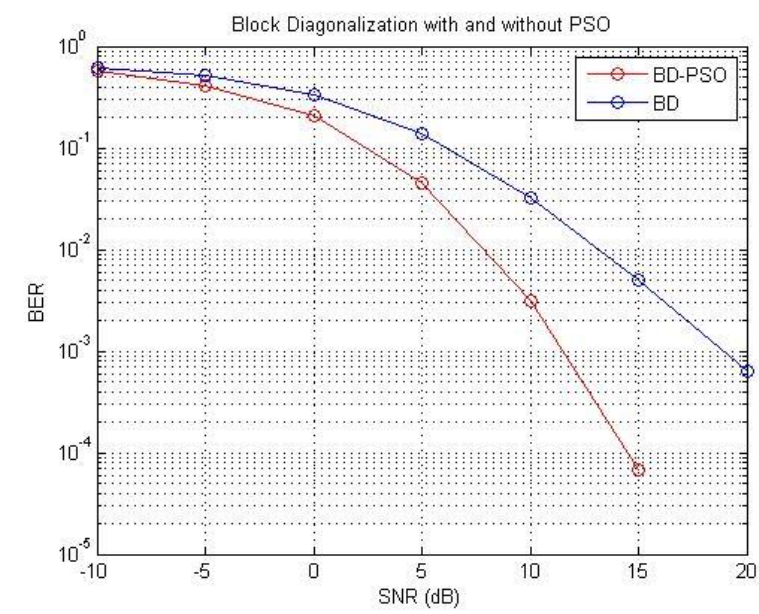

Fig. 4: BER curve for BD and PSO optimized BD

\section{Conclusion}

Multiple-Input Multiple Output (MIMO) technology is a promising solution to several problems of modern communication scenario including high data rate requirement and reliability. The major disadvantage of MIMO system is their complexity. The performance of MIMO system can be enhanced using application of Channel State Information (CSI) at transmitter end. One solution to the above problem is use precoding technique at the transmitter end. Block-Diagonalization is a popular precoding technique and it has proven performance in Multi-User scenario (MUMIMO) as well. The major drawback of Block Diagonalization scheme is that it requires more antennas at receiver end as compared to the antennas mounted on transmitter end. The search of optimal antenna arrangement with required performance is a tiresome work. Recently evolutionary algorithms have found application in communication system designs. The problem of optimal number of antenna selection can also be solved through evolutionary algorithms. In this regard, this research work presents optimization of block-diagonalization precoding scheme using PSO for MU-MIMO downlink system. Simulation scenario is developed using MATLAB (2014a) and the developed system is simulated over Rayleigh fading environment. To evaluate performance of developed system scenario Bit Error Rate (BER) is considered. Simulation results validate that the performance of Block- Diagonalization scheme can be improved up to $5 \mathrm{~dB}$ with the application of Particle Swarm Optimization technique.

\section{References}

[1] Foschini, Gerard J. "Layered space-time architecture for wireless communication in a fading environment when using multi-element antennas." Bell labs technical journal 1.2 (1996): 4159.

[2] Telatar, Emre. "Capacity of Multi-antenna Gaussian Channels." Transactions on Emerging Telecommunications Technologies 10.6 (1999): 585-595.

[3] H. Sampath, P. Stoica, and A. Paulraj. Generalized linear precoder and decoder design for MIMO channels using the weighted MMSE criterion. IEEE Trans. Commun., 49(12) :21982206, 2001

[4] Tsoulos, George, ed. MIMO system technology for wireless communications. CRC press, 2006.

[5] Caire, Giuseppe, and ShlomoShamai. "On the achievable throughput of a multiantenna Gaussian broadcast channel." IEEE Transactions on Information Theory 49.7 (2003): 1691-1706.

[6] Jindal, Nihar, and Andrea Goldsmith. "Dirty-paper coding versus TDMA for MIMO broadcast channels." IEEE Transactions on Information Theory 51.5 (2005): 1783-1794.

[7] Stankovic, Veljko, and Martin Haardt. "Successive optimization Tomlinson-Harashima precoding (SO THP) for multi-user MIMO systems." Acoustics, Speech, and Signal Processing, 
2005. Proceedings.(ICASSP'05). IEEE International Conference on. Vol. 3. IEEE, 2005.

[8] Liavas, Athanasios P. "Tomlinson-Harashima precoding with partial channel knowledge." IEEE Transactions on Communications 53.1 (2005): 5-9.

[9] Peel, Christian B., Bertrand M. Hochwald, and A. Lee Swindlehurst. "A vector-perturbation technique for near-capacity multiantenna multiuser communication-part I: channel inversion and regularization." IEEE Transactions on Communications 53.1 (2005): 195-202.

[10] Shaik Yasmin Fathima, Md. Zia Ur Rahman, K. Murali Krishna, Shakira Bhanu,Mirza Shafi, "Side Lobe Suppression in NCOFDM Systems Using Variable Cancellation Basis Function", IEEE Access, vol.5, no.1, pp. 9415-9421, 2017.

[11] Spencer, Quentin H., A. Lee Swindlehurst, and Martin Haardt. "Zero-forcing methods for downlink spatial multiplexing in multiuser MIMO channels." IEEE transactions on signal processing 52.2 (2004): 461-471.

[12] Wang, X. "Robust THP design with the presence of erros in CSIT acquisition." China Communications 7.2 (2010): 78-86.

[13] Haardt, Martin, VeljkoStankovic, and Giovanni Del Galdo. "Efficient multi-user MIMO downlink precoding and scheduling." Proc. IEEE Int'l Work. Comp. Advances in Multi-Sensor Adaptive Processing, Puerto Vallarta, Mexico (2005).

[14] Stankovic, Veljko, and Martin Haardt. "Multi-user MIMO downlink precoding for users with multiple antennas." Proc. of the 12 th Meeting of the Wireless World Research Forum (WWRF), Toronto, ON, Canada. Vol. 10. 2004.

[15] Wang, Feng, and Marek E. Bialkowski. "Performance of multiuser MIMO system employing block diagonalization with antenna selection at mobile stations." Signal Processing Systems (ICSPS), 2010 2nd International Conference on. Vol. 1. IEEE, 2010.

[16] Naeem, Muhammad, and Daniel C. Lee. "A joint antenna and user selection scheme for multiuser MIMO system." Applied Soft Computing 23 (2014): 366-374.

[17] Ni, Weiheng, and Xiaodai Dong. "Hybrid block diagonalization for massive multiuser MIMO systems." IEEE transactions on communications 64.1 (2016): 201-211.

[18] Zhu, Xudong, et al. "Soft pilot reuse and multicell block diagonalization precoding for massive MIMO systems." IEEE Transactions on Vehicular Technology 65.5 (2016): 3285-3298.

[19] Chen, Jung-Chieh, et al. "Low-complexity precoding design for massive multiuser MIMO systems using approximate message passing." IEEE Transactions on Vehicular Technology 65.7 (2016): 5707-5714.

[20] Crâşmariu, Victor-Florin, et al. "Optimized blockdiagonalization precoding technique using givens rotations $\mathrm{QR}$ decomposition." Signal Processing Conference (EUSIPCO), 2017 25th European. IEEE, 2017.

[21] Nayebi, Elina, et al. "Precoding and power optimization in cellfree massive MIMO systems." IEEE Transactions on Wireless Communications 16.7 (2017): 4445-4459.

[22] Ge, Mengyao, and Douglas M. Blough. "PBUS: Efficient User Selection for Block Diagonalization in Dense Wireless Networks." GLOBECOM 2017-2017 IEEE Global Communications Conference. IEEE, 2017.

[23] Sindhwani, Nidhi, et al. "Performance analysis of particle swarm optimization and genetic algorithm in MIMO systems." Computing, Communication and Networking Technologies (ICCCNT), 2017 8th International Conference on. IEEE, 2017.

[24] Chen, Jung-Chieh. "Low-PAPR precoding design for massive multiuser MIMO systems via Riemannian manifold optimization." IEEE Communications Letters 21.4 (2017): 945-948.

[25] Makki, Behrooz, et al. "A genetic algorithm-based antenna selection approach for large-but-finite MIMO networks." IEEE Transactions on Vehicular Technology 66.7 (2017): 6591-6595.

[26] Telatar, E. (1999). Capacity of Multi-antenna Gaussian Channels. Transactions on Emerging Telecommunications Technologies, 10(6), 585-595.

[27] Cao, Bin, et al. "PEACE: Polarization enabled active cooperation scheme between primary and secondary networks." IEEE Transactions on Vehicular Technology 63.8 (2014): 3677-3688. 